\title{
Case 1,2012: A 65 Years Old Man with Tetanus
}

\section{Tarik I Zaher ${ }^{1}$, Khaled M Helmy ${ }^{2}$}

${ }^{I}$ Tropical Medicine Depatrment, Faculty of Medicine, Zagazig University, Egypt

${ }^{2}$ Anesthesia Department and Intensive Care Unit, Faculty of Medicine, Zagazig University ,Egypt tareqzaher@zu.edu.eg

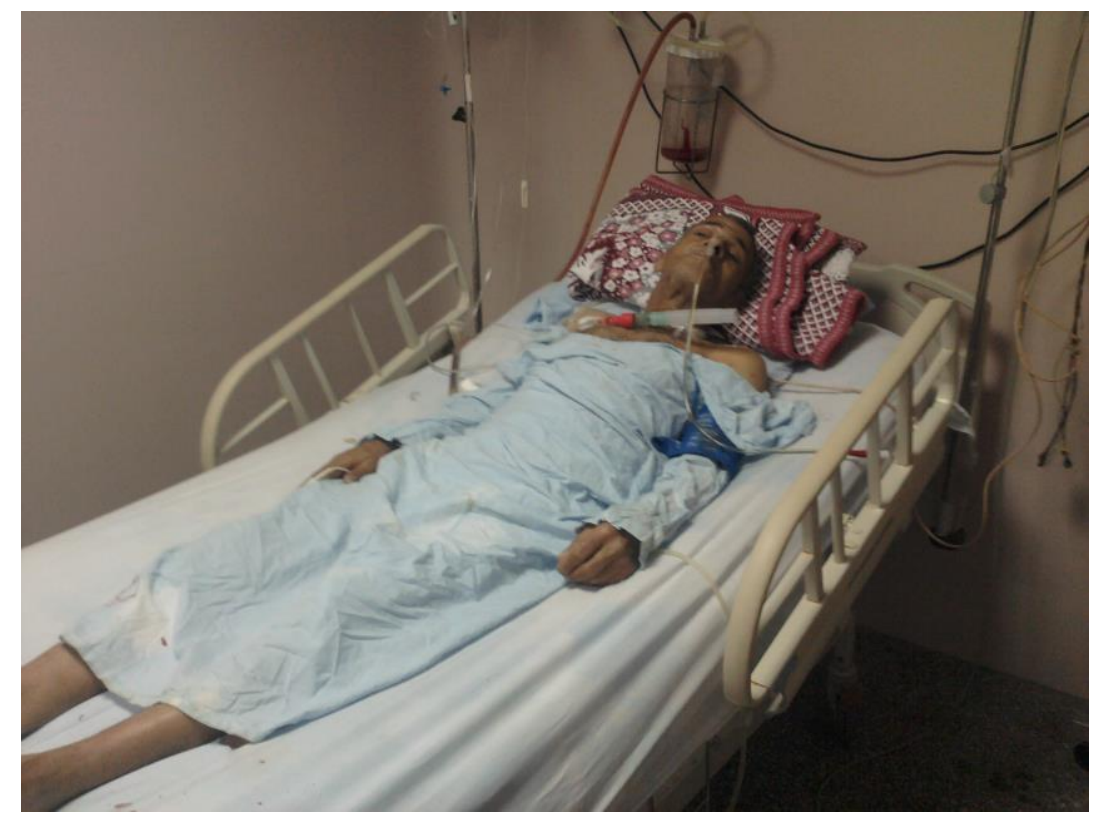

\section{Presentation of the case:}

A 65 years old man from Dyrab Nejm, Sharkia Governorate, Egypt with wound in the foot due to penetration by a nail was presented 2 weeks later by dysphagia, trismus (lock jaw), rigidity of the neck, limbs and abdominal muscles. There was no history of bite. Attacks of spasms (convulsions) with occasional cyanosis were noticed. The patients was admitted initially to Zagazig Fever Hospital, then the patient was referred to Intensive Care Unit, Surgical Building, Zagazig University Hospitals, CSF examination was normal, tracheostomy was performed with aspiration of chest secretions and oxygen supply through it. Nasogastric tube (Ryle) was also difficulty introduced. The feeding was enteral .100,000 U equine antitetanic serum was administered intramuscularly in the first days of hospitalization after test for hypersensitivity . The patient was conscious with inability to talk. He wrote what he wanted. The patient was put in dark silent room. Diazepam in a dose of 20 $\mathrm{mg} / 8 \mathrm{hrs}$ infusion was administered for 3 weeks then tapered gradually. Intravenous muscle relaxants was administered for 4 weeks also chlorpromazin in a dose of $50 \mathrm{mg} / 8 \mathrm{hrs}$ through the Ryle was administered in the first 2 weeks. Metronidazol 500 I.V. every $8 \mathrm{hrs}$ for 10 days was administered as well as prophylactic macrolid antibiotic. By the end of 4 th week of illness the spasms ceased and the patient could talk but neck, abdominal and limbs rigidity were still present .

\section{Differential diagnosis[1,2]:}

1. Local causes of trismus as dental abscess, mumps and quinzy.

2. Meningitis and encephalitis.

3. Tetany.

4. Strychnine poisoning.

5. Peritonitis. 
6. Rabies.

7. Epilepsy.

8. Dystonic reaction to phenothiazin.

\section{Discussion:}

Tetanus still continues to constitute a serious health problem in Egypt and other developing countries. The caustive organism is clostridium tetani which is Gram-positive rod shaped bacteria. Spores are present in soil ,street dust, human and animal feces. Two exotoxins are liberated by the organisms namely tetanospasmin and tetanolysin. Tetanospasmin increases the excitability of the spinal cord and has also a local action on the muscles[1].

The above case is a typical case of tetanus due to presence of lock jaw, rigidity of muscles, tonic spasms and absence of disturbance of consciousness (conscious till the end).

Local causes of trismus are excluded due to absence of neck lymph nodes and presence of other features of tetanus as generalized muscle rigidity and spasms.

Meningitis and encephalitis are excluded due to normal CSF examination and presence of generalized muscle rigidity rather than only neck rigidity of meningitis.

In tetany the spasms are confined to the hands and feet, so it is excluded.

No history of ingestion of strychnine, so it is excluded.

In peritonitis the rigidity is confined to the abdomen.

Early stages of rabies (Hydrophobia) may be confused with dysphagia of tetanus. However no rigidity of muscles are present in rabies.
Epilepsy is associated with clonic convulsions rather than tonic spasms of tetanus.

The patient survived due to early introduction of antitetanic serum, good intensive care and tracheostomy with aspiration of pulmonary secretions and introduction of prophylactic antibiotics.

Tetanus has a 40-60\% mortality due to pneumonia, respiratory failure, circulatory disturbance and septicemia[2].

Puncture wounds like this case, animal and human bites, wounds contaminated with soil or feces and burns should not be sutured and cleaned. A booster dose of tetanus toxid should be given if the last dose was given 5-10 years ago. If no active immunization was given or given more than 10 years ago, a full dose of tetanus toxid is given with passive immunization by 250 units intramuscularly of human tetanus immunoglobulines (HTIG) or 1500 units equine antitetanic serum. The dose should be doubled in high risk or delayed wounds [2].

\section{Conclusion:}

Tetanus is still a health problem in developing countries as Egypt. Intensive care of tetanus patient and early antitetanic serum as well as tracheostomy and mechanical ventilation if needed decrease the mortality .

\section{References:}

1. Saif El Din S and Abdel Wahab MF. A Guide Book of Tropical and Infectious Diseases. Tropical Medicine Department, Ain Shams University , Cairo 1995;244-246.

2. Smith MD. Tetanus .Cook GC ,ed .Manson's Tropical Diseases .Saunders, 20th edition 1996; 48:906-913. 